\title{
SPR sensor for detection of heavy metal ions: Manipulating the EM waves polarization modes
}

\author{
Wan Maisarah Mukhtar a, ${ }^{*}$, Razman Mohd Halim ${ }^{\text {b }}$, Karsono Ahmad Dasuki ${ }^{a}$, Affa Rozana Abdul \\ Rashid ${ }^{\mathrm{a}}$, Nur Athirah Mohd Taib ${ }^{\mathrm{a}}$ \\ a Faculty of Science and Technology, Universiti Sains Islam Malaysia (USIM), Bandar Baru Nilai, Negeri Sembilan, Malaysia; \\ ${ }^{b}$ National Metrology Institute of Malaysia, Bandar Baru Salak Tinggi, Sepang, Malaysia. \\ *Corresponding author: wmaisarah@usim.edu.my
}

\section{Article history}

Received 7 September 2017

Accepted 5 November 2017

\begin{abstract}
The development of high sensitivity surface plasmon resonance (SPR) sensor depends on few crucial factors such as types and thicknesses of metal thin films, light coupling techniques and suitable polarization modes of EM waves. This work was carried out to investigate the effect of EM wave polarization modes on the sensing properties of SPR sensor in detecting heavy metal ions namely mercury $(\mathrm{Hg})$ and plumbum $(\mathrm{Pb})$. Three types of light polarization modes such as $\mathrm{p}$ polarized, s-polarized and circular-polarized light were introduced. Gold and silver thin films with thicknesses of $50 \mathrm{~nm}$ were deposited on top of SPR layer system to excite surface plasmon polaritons (SPPS). The SPR curves were analyzed by studying the FWHM, Q-factor and angle shifting characteristics. We managed to prove theoretically that the SPR phenomena able to be created by using not only the p-polarized light, yet by employing a circular-polarized light. The sensor showed positive respond for both polarization modes, where the troughs were red-shifted about $23.78 \%$ as $\mathrm{Pb}$ was introduced on the top of the gold-coated SPR sensor. The SPR angles were shifted about $24.45 \%$ as the sensor was exposed with $\mathrm{Hg}$ metal ions. Different response due to the presence of different analytes exhibited excellent criteria of high selectivity sensor. In conclusion, the combination of $p$-polarized light or circular-polarized light and gold thin film able to accentuate the significant role of SPR sensor in detecting heavy metal ions.
\end{abstract}

Keywords: SPR sensor, EM waves polarization modes, heavy metal ions, optical sensor

\section{INTRODUCTION}

Water pollution is contamination of water by foreign matter that reduce the quality of water. Most of the pollutants such as lead $(\mathrm{Pb})$, arsenic (As) and mercury (Hg) are very harmful (Verma et al., 2013) Water contamination by heavy metals is a common issue encountered in many countries due to industrial and urban wastes in water resources which give health and socio-economic impacts (Borah et al., 2014). There are several methods have been introduced to detect the presence of heavy ions metals such as enzyme sensor, electrochemical sensor, piezoelectric sensor etc (Bagal et al., 2008; Cui et al., 2008; Ghica et al., 2008; Huang et al., 2007). However, there are few weaknesses of these methods such as sophisticated procedure and costly. The development of suitable sensors with high responsivity, excellent sensitivity and and less expensive are increasingly becoming a priority.

Today, the developments of optical sensors are growth rapidly. The demand of fiber optics sensors (Verma et al., 2015; Zhao et al., 2013) and free space optics sensors (Li et al,. 2014; Nizamiv et al. 2015) indicate the society's acceptance to these technologies. Nonetheless, the applications for each sensor are limited to certain applications such as for gas sensing (Viter et al., 2015), liquid sensing (Wang et al., 2014), chemical sensing (Askim et al., 2013) etc. In recent years, Surface Plasmon Resonance (SPR) is one of the common technique for sensing purpose. SPR phenomena involving the interaction of matter and light. It occurs when the polarized light which is under total internal reflection (TIR) conditions strikes the metal thin film layer with dielectric negative that has been placed at the interfaces between two dielectric media of different refractive angle through the prism (Mukhtar et al., 2013).

SPR sensor is one of the favourable optical sensors due to its high sensitivity (Murat et al., 2016; ). Many works have been conducted to optimize the SPR signals by controlling the metal's thicknesses, introducing semiconductor materials and depositing metal nanoparticles. Critical factors that need to be addressed is to ascertain the stability and the accuracy of SPR sensor system itself. Based on previous studies (Mukhtar et al., 2014; Liu et al., 2016), light polarization mode is one of the main criteria that need to be obeyed to generate SPR. The main objective of this work is to study the effect of EM wave polarization modes on the sensing properties of SPR sensor in detecting heavy metal ions. By fixing the control parameters such as properties of metal thin films, light polarization modes and dielectric constants of analytes; the characteristics of SPR curves were investigated. 


\section{EXPERIMENTAL}

\section{Methods}

A red laser with excitation wavelength of $633 \mathrm{~nm}$ was employed as a light source. Types of polarization modes were varied as ppolarized, s-polarized and circular-polarized. The existence of Brewster angle and location of critical angle were investigated by observing the behavior of reflected light from a bare prism. The appearance of Brewster angle is an important indicator for the excitation of surface plasmon polaritons (SPPs) (Mukhtar et al., 2013a). The values of incident angles were modulated from $38^{\circ}$ to $70^{\circ}$. For excitation of SPR signals, two types of metal thin films namely silver (dielectric constant, $\varepsilon_{\mathrm{s}}=-17+0.7 \mathrm{i}$ ) and gold (dielectric constant $\varepsilon_{\mathrm{g}}=-9.39+1.09 \mathrm{i}$ ) with thicknesses of $50 \mathrm{~nm}$ were appointed. By tuning the angle of light incidence of the totally reflected beam inside the prism, the resonance condition for excitation of SPPs can be fulfilled (Novotny et al., 2006). Figure 1 displays an example of SPR experimental setup for detection of the targeted analytes (Mukhtar et al., 2013b). To observe the significant role of SPR sensor in detecting the heavy metal ions, plumbum $(\mathrm{Pb})$ and mercury $(\mathrm{Hg})$ with values of dielectric constant $\varepsilon_{\mathrm{Pb}}=3.5344$ and $\varepsilon_{\mathrm{Hg}}=3.9026$ respectively, were introduced on top of metal thin films. Table 1 tabulates the values and properties of four-layer system of SPR sensor. The important parameters such as Q-factor, full width half maximum (FWHM) and angle shifting were analyzed.

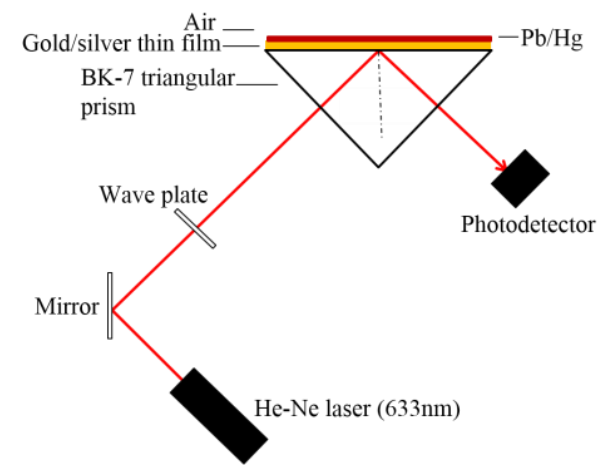

Fig. 1 Illustration of SPR experimental setup for heavy metal ions $(\mathrm{Pb} / \mathrm{Hg})$ detection. The function of optical waveplate is to modulate the polarization mode of EM waves.

Table 1 The four-layer system of SPR sensor.

\begin{tabular}{cclccc}
\hline \multirow{2}{*}{ Layer } & & Types & \multirow{2}{*}{$\begin{array}{c}\text { Thickness, } t \\
(\mathrm{~nm})\end{array}$} & \multicolumn{2}{c}{ Value of dielectric constant, $\varepsilon$} \\
\cline { 5 - 6 } & & & neal part & Imaginary part \\
\hline \hline 1 & Prism & dielectric & none & 2.29 & 0 \\
\hline 2 & \multirow{2}{*}{ Metal } & gold & 50 & -9.39 & 1.09 \\
& silver & 50 & -17 & 0.7 \\
\hline \multirow{2}{*}{3} & Heavy & Plumbum $(\mathrm{Pb})$ & none & 3.5344 & 0 \\
& metal & & & & \\
& ions & Mercury $(\mathrm{Hg})$ & none & 3.9026 & 0 \\
\hline 4 & Air & dielectric & none & 1 & 0 \\
\hline
\end{tabular}

\section{RESULTS AND DISCUSSION}

Figure 2(a) depicts the location of critical angle as light polarization modes were set as p-polarized, s-polarized and circular polarized. It was found that the critical angles situated at $39.5^{\circ}$ only existed for p-polarized and circular polarized modes, in which the total internal reflection (TIR) phenomena were occurred. It is important to monitor the position of critical angle because this value can be used as the location's indicator of SPR angle. The location of SPR angle is always within the range of TIR (Novotny et al., 2006). In this work, the location of SPR angles were expected to be above $39.5^{\circ}$. Below this angle, SPR cannot occur due to the insufficient energy provided by the incident light (Novotny et al., 2006). About 91.93\% of the incident light experienced TIR as it was set under p- polarization mode. As the mode was changed into circularlypolarized, $60 \%$ of incident light was totally reflected. Those percentage amounts of reflected light will be consumed to generate SPPs. Obviously, the TIR phenomena cannot be established as the polarization mode was designated as s-polarized. The presence of Brewster angle (circle-dashed line) also can be used as an indicator for the existence of SPR phenomena (Mukhtar et al., 2013a). It was found that only p-polarized and circular polarized light indicated the appearances of Brewster angles at $27.5^{\circ}$ as captured in Figure 2(b).

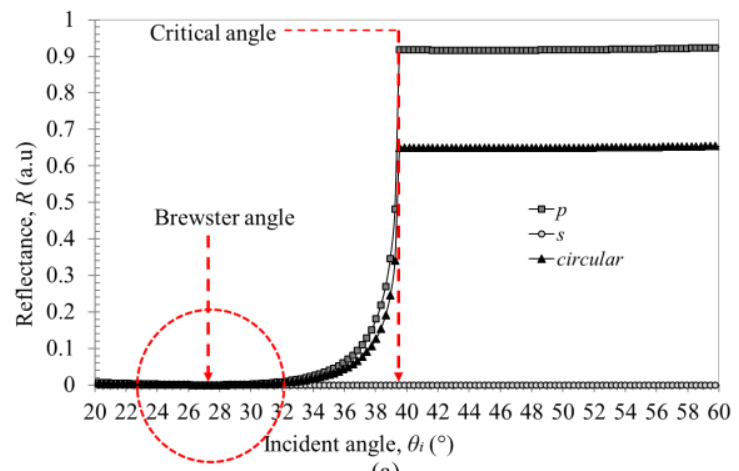

(a)

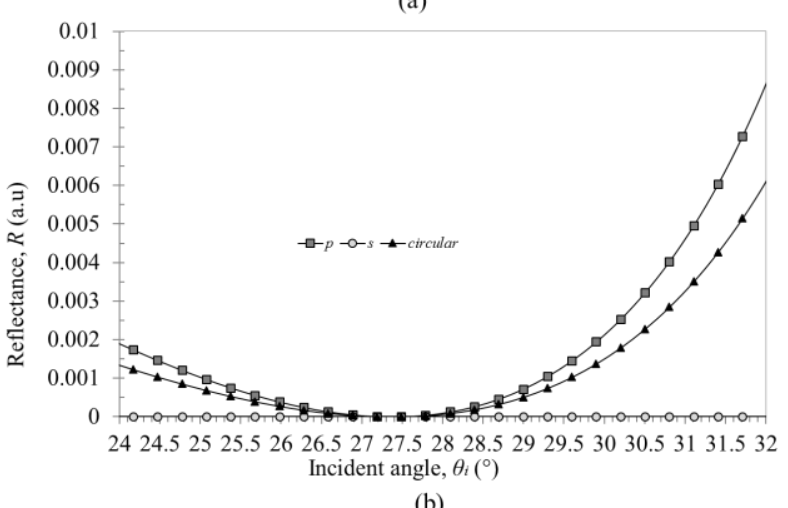

(b)

Fig. 2 (a) Location of critical angle and Brewster angle using triangular prism with refractive index of 1.51 for various light polarization, (b) Zoom-in the location of Brewster angle from Figure 2(a).

Figure 3 displays the SPR curves as gold thin films were coated on the glass substrate. Minimum values of $\mathrm{R}=0$ for three types of polarization modes were obtained. Note that the justification of strong SPR signals were not genuinely represented by the value of minimum reflectance; to be exact, the SPR curve depth or Q-factor analyses able to explain this properties. Obviously, s-polarization mode resulted the absence of SPR signal which indicates an excellent agreement with Figure 2. This result clarified the crucial role of Brewster angle in determining an appropriate polarization mode for the establishment of SPR. In comparison between circular and p-polarized modes of incident light with the usage of gold thin film to excite SPPs, (Figure $3(a)$ ), the circular polarization mode resulted weaker SPR signal than p-polarized where only $60.23 \%$ of the incident light were excited as SPPs, meanwhile about $85.33 \%$ of the incident light were generated as SPPs for p-polarized light. The FWHM value of $2.931^{\circ}$ for $\mathrm{p}$ polarization mode was smaller than the circular mode. For circular polarized light, the FWHM was obtained as $3.1034^{\circ}$.

As type of thin film was replaced with silver, the values of FWHM were drastically reduced by observing the decrease of SPR curves' width. The values of FWHM of p-polarized and circular polarized modes were nearly the same which are $\mathrm{FWHM}_{p}$. polarized $=0.25^{\circ}$ and $\mathrm{FWHM}_{\text {circular polarized }}=0.23^{\circ}$. As expected, same characteristics of light reflectance behaviour were observed where no SPR signal was produced as s-polarized light was appointed. Strong signals about $89.60 \%$ was resulted with the employment of ppolarized light and $63.35 \%$ for circular polarized light. According to these results, it can be summarized that SPPs can be excited by manipulating the light polarization modes either into p-polarized or circular polarized. s-polarized mode happens to result no SPR signal 
by considering the characteristics of EM waves where the oscillation of electrons are on the same direction with the glass plate, meanwhile for p-polarized light the SPPs were perpendicularly oscillated on the glass surface where the SPR signals can easily be observed (Saleh et al., 1991).

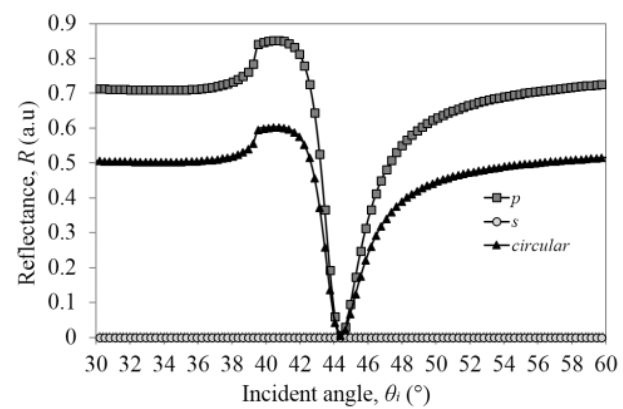

(a)

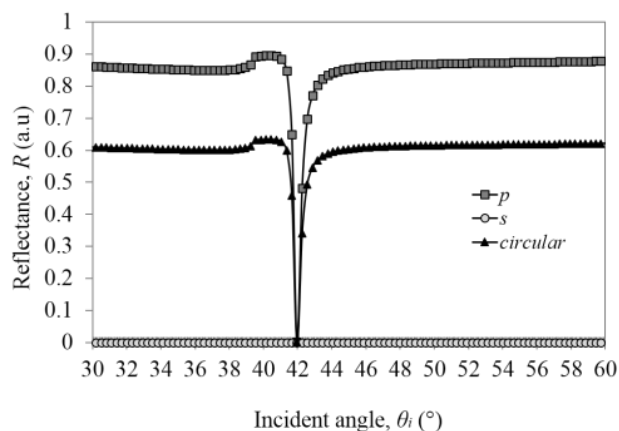

Fig. 3 SPR curves for several types of polarization modes namely $p$ polarized, s-polarized and circular polarized by using (a) gold thin film (b) silver thin film.

To study the role of light polarization modes on the sensitivity of SPR sensor, two types of analytes were introduced on top of metal thin film, namely mercury $(\mathrm{Hg})$ and plumbum $(\mathrm{Pb})$. Figure $4(\mathrm{a})$ displays the characteristics of SPR signals with the employment of gold thin film. The sensor showed positive respond for both polarization modes, where the curves were red-shifted about $23.78 \%$ from $44.37^{\circ}$ to $54.92^{\circ}$ as $\mathrm{Pb}$ was introduced on the top of the sensor. For p-polarization mode, the FWHM value was increased about $73 \%$ to $10.855^{\circ}$ when the SPR sensor reacted with $\mathrm{Pb}$. As the polarization mode was changed into
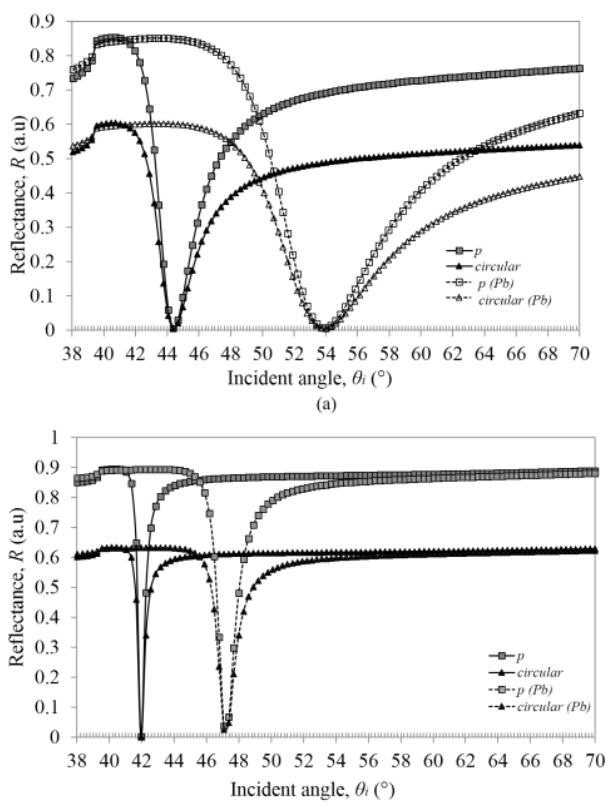

(b)

Fig. 4 SPR signal responds with the presence of $\mathrm{Pb}$ ions (a) gold thin film (b) silver thin film. circular mode, the FWHM was obtained as $10.560^{\circ}$ with $70.61 \%$ of increment in comparison with the absence of analyte. The influence of metal thin film's types on the sensor sensitivity was investigated by replacing gold thin film with silver as captured in Figure 4(b). The SPR angle was shifted about $12.22 \%$ from $41.96^{\circ}$ to $47.09^{\circ}$. The observation of angle shifting exhibits the ability of SPR sensor to detect the presence of $\mathrm{Pb}$ metal ions. This output shows that gold results better sensitivity than silver due to its strong response. The properties of silver which are reactive and easily oxidized contribute to the less sensivity performance of SPR sensor (Akowuah et al., 2012).

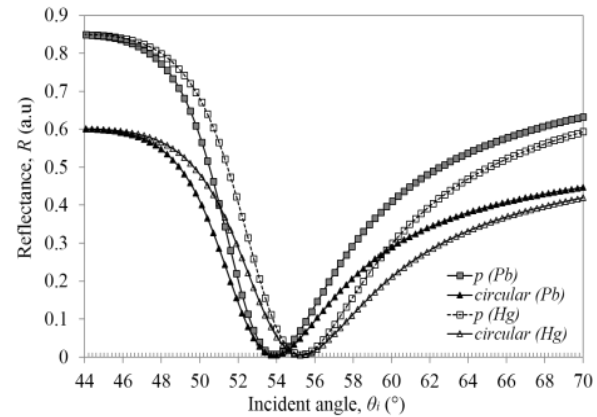

(a)

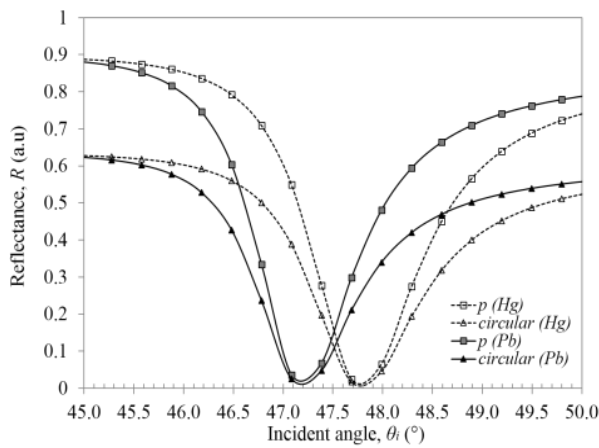

(b)

Fig. 5 SPR signal responds for the detections of $\mathrm{Hg}$ and $\mathrm{Pb}$ metal ions with employments of (a) gold thin film (b) silver thin film.

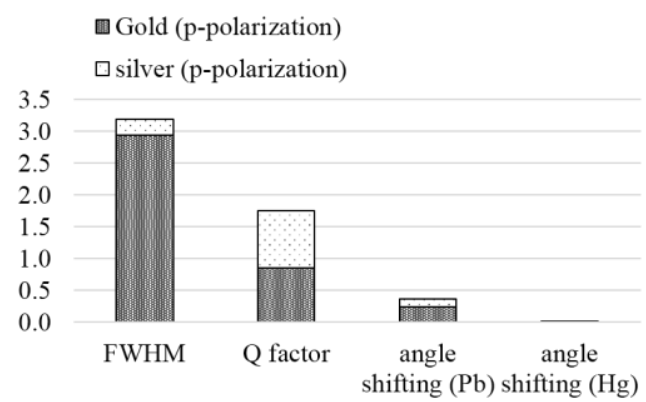

(a)

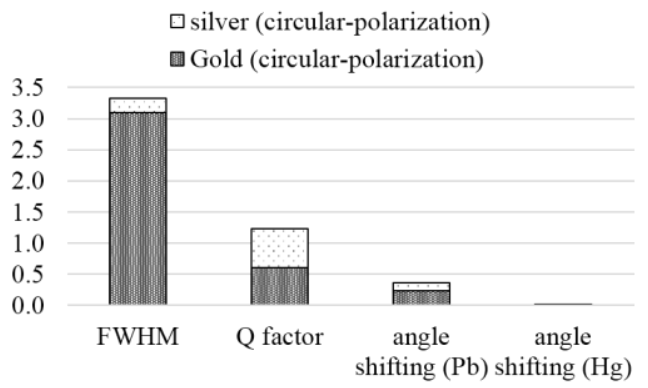

(b)

Fig. 6 Parameter analyses on the performance sensitivity of SPR sensor using (a) p-polarized light (b) circular polarized light. 
One of the important features of an excellent potential optical sensor is its ability to differentiate various types of analytes or can be simplified as selectivity. To achieve this, we compare the SPR signal response as $\mathrm{Hg}$ and $\mathrm{Pb}$ metal ions were introduced on top of the sensor. Figure 5(a) shows the angle shifting experienced by SPR sensor embedded with gold thin film as different analytes were added on top of the sensor. As $\mathrm{Pb}$ was replaced with $\mathrm{Hg}$, the SPR angles were shifted about $0.5 \%$ from $54.92^{\circ}$ to $55.22^{\circ}$. However, types of polarization mode, neither p-polarized nor circularly polarized did not result any impact on SPR angle shifting where both modes experienced same amount of shifting. By substituting $\mathrm{Hg}$ metal ions, the SPR curves experienced $1.27 \%$ of shifting for silver thin film as depicted in Figure 5(b).

Figure 6 summarized the analyses parameters on the sensitivity performance of SPR sensor. The analysis of FWHM between $p$ polarized and circular-polarized portrayed similar behaviour where large FWHM was obtained by employing gold thin film. Q-factor analysis indicated that the usage of p-polarized light with the assistance of gold thin film for SPPs generation produced deeper SPR curve depth than circular-polarized which explains the cability of these combinations to yield strong SPR phenomena. In comparison with silver, the development of high sensitivity SPR sensors can be achieved by employing $50 \mathrm{~nm}$ gold thin film where apparent angle shifting was observed as heavy metal ions were introduced on the sensors. The ability in detecting different types of analytes namely $\mathrm{Pb}$ and $\mathrm{Hg}$ based on amount of angle shifting indicates an impressive selectivity features of gold-coated SPR sensor.

\section{CONCLUSION}

This study investigates few crucial parameters which affect the performance of SPR sensors by controlling type of light polarization modes. It was found that the Q-factor values which represents by the SPR curve depth plays a significant role in achieving excellent SPR sensor. The major finding of this work proves that the SPR phenomena able to be created with the usage of circular-polarized light, apart from p-polarized light. In conclusion, the combination of EM wave p-polarization mode and gold thin film able to accentuate the significant role of SPR sensor in detecting heavy metal ions.

\section{ACKNOWLEDGEMENT}

The work was supported by Universiti Sains Islam Malaysia and Ministry of Higher Education (MOHE) under grant FRGS/2/2014/SG02/USIM/03/1. Knoll Group from Max Planck Institute for Polymer Research, German is acknowledged for the Winspall 3.02 simulation software.

\section{REFERENCES}

Akowuah, E. K., Gorman, T., Ademgil, H. and Haxha, S. 2012. A highly sensitive photonic crystal fibre (PCF) surface plasmon resonance (SPR) sensor based on a bimetallic structure of gold and silver. 2012 IEEE 4th International Conference on Adaptive Science \& Technology (ICAST). 2527 October 2012. Kumasi, Ghana: IEEE, 121-125.

Alwahib, A. A., Sadrolhosseinic, A. R., Limd, H. N., Yaacob, M. H., Bakar, M. A. and Mahdi, M. A. 2016. Study of EDC/NHS immobilization for plumbous detection using surface plasmon resonance. Jurnal Teknologi, 78(3), 253-256

Askim, J. R., Mahmoudi, M., Suslick, K. S. 2013. Optical sensor arrays for chemical sensing: the optoelectronic nose. Chemical Society Reviews, 42, 8649-8682.

Bagal-Kestwal, D., Karve, M. S., Kakade, B., Pillai, V. K. 2008. Invertase inhibition based electrochemical sensor for the detection of heavy metal ions in aqueous system: Application of ultra-microelectrode to enhance sucrose biosensor's sensitivity. Biosensors and Bioelectronics, 24(4), $657-$ 664 .

Borah, S. B. D., Baruah, S., Mohammed, W. S., Dutta, J. 2014. Heavy Metal Ion Sensing By Surface Plasmon Resonance on Gold Nanoparticles. ADBU Journal of Engineering Technology. 1-5.

Cui, L., Wu, J., Ju, H. 2015. Electrochemical sensing of heavy metal ions with inorganic, organic and bio-materials. Biosensors and Bioelectronics, 63, 276-286.
Ghica, M. E.; Brett, C. M. 2008. Glucose oxidase inhibition in poly (neutral red) mediated enzyme biosensors for heavy metal determination. Microchimica Acta. 163(3-4), 185-193.

Huang, G. S., Wang, M. T., Su, C. W., Chen, Y. S., Hong, M. Y. 2007. Picogram detection of metal ions by melanin-sensitized piezoelectric sensor. Biosensors and Bioelectronics, 23(3), 319-325.

Li, Y., Yan, H., Farmer, D. B., Meng, X., Zhu, W., Osgood, R. M., et al. 2014. Graphene plasmon enhanced vibrational sensing of surface-adsorbed layers. Nano Letters, 14, 1573-1577.

Liu, C., Liu, Q., Qin, Z. 2016. Determination of the Bimetallic Layers' Film Thicknesses by Phase Detection of SPR Prism Coupler. Plasmonics. 1-6. Mukhtar, W. M., Shaari, S., Menon, P. S. 2013a. Influences of light coupling techniques to the excitation of surface plasmon polaritons. Advanced Science Letters, 19, 66-69.

Mukhtar, W. M., Shaari, S., Menon, P. S. 2013b. Propagation of surface plasmon waves at metal thin film/air interface using modified optical waveguiding assembly. Journal of Optoelectronics and Advanced Materials, 7, 9-13.

Mukhtar, W. M., Shaari,. S, Ehsan, A. A., Menon, P. S. 2014. Electro-optics interaction imaging in active plasmonic devices. Optical Materials Express, 4, 424-433.

Murat, N. F., Mukhtar, W. M., Rashid, A. R. A., Dasuki, K. A., Yussuf, A. A. R. A. 2016. Optimization of gold thin films thicknesses in enhancing SPR response. International Conference on Semiconductor Electronics (ICSE), 2016 Aug 7; Kuala Lumpur. p. 244-247.

Nizamov, S., Scherbahn, V., Mirsky, V. M. 2015. Self-referencing SPR-sensor based on integral measurements of light intensity reflected by arbitrarily distributed sensing and referencing spots. Sensors and Actuators B: Chemical, 207, 740-747.

Novotny, L., Hecht, B. 2006. Principles of Nano-Optics. United Kingdom (UK): Cambridge University Express.

Saleh, B. E. A., Teich, M. C. 1991. Fundamentals of Photonics. United States (US): John Wiley \& Sons Inc.

Verma, R., Dwivedi, P. 2013. Heavy metal water pollution-A case study. Recent Research in Science and Technology. 5, 98-99.

Verma, R., Gupta, B. D. 2015. Detection of heavy metal ions in contaminated water by surface plasmon resonance based optical fibre sensor using conducting polymer and chitosan. Food Chemistry, 166, 568-575.

Viter, R., Chaaya, A. A., Iatsunskyi, I., Nowaczyk, G., Kovalevskis, K., Erts, D., et al. 2015. Tuning of $\mathrm{ZnO} 1 \mathrm{D}$ nanostructures by atomic layer deposition and electrospinning for optical gas sensor applications. Nanotechnology. 26, 105501-

Wang, W., Li, F. 2014. Large-range liquid level sensor based on an optical fibre extrinsic Fabry-Perot interferometer. Optics and Lasers in Engineering, 52, 201-205.

Zhao, Q., Yin, M., Zhang, AP., Prescher, S., Antonietti, M., Yuan, J. 2013. Hierarchically structured nanoporous poly (ionic liquid) membranes: Facile preparation and application in fiber-optic $\mathrm{pH}$ sensing. Journal of the American Chemical Society, 135, 5549-5552. 